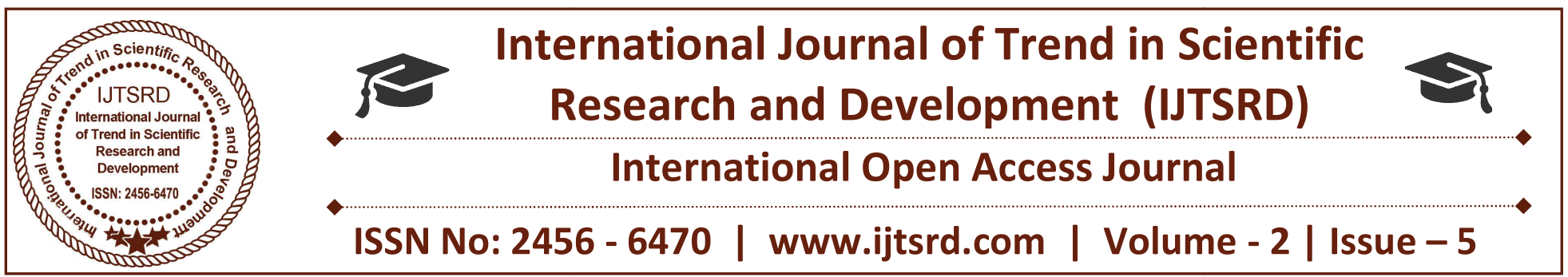

\title{
Nanorobots in Cancer Treatment
}

\author{
Santhiya Grace. A, Devi Kala Rathinam. D, Sherin. J \\ PG Scholar \\ Department of Computer Science and Engineering, \\ Sri Krishna College of Engineering and Technology, \\ Coimbatore, Tamil Nadu, India
}

\begin{abstract}
Nanorobotics is one of the emerging and advanced technology in various field. The scientist throughout the world involves in research and development of nanorobots. The size of nanorobots is 10-9 meter and it is opposed to microrobotics. It is composed of many nano components. The range of device is 0.1-10 micrometer.It plays main role in medical industry as well as space industry. Nano medicine is developed using nanorobots. The nanorobots is highly durable. The largest contribution is given by IT (Information
\end{abstract} Technology) and sensor Technology for the development of Nano Technology. Nanorobots is different from macro robots due to some scale and material, even there are some similarities in the design and control techniques. The nanorobots is called as self- replication because it replace worn-out units by producing the copies of themselves. Nanorobots helps to treat some diseases like removal of kidney stone, diagnose and treat the cancer cells in human body, eliminate the defected parts in DNA, cerebral Aneurysm and also some other treatments and save the life of human. Robotic devices are used to help the patient in hospital rooms, complex surgery can also be performed using the robot, laboratory test can also be performed, some missing limbs can be replaced, help in rehabilitation after stroke and also to diagnose some diseases. Nanorobots are nano devices used for protecting and maintaining the human body against pathogens. Nanorobotics can be named as nanoids, nonomites, nanites or nanorobots can be used to describe hypothetical devices. This paper helps to review the working of nanorobots in medical field and also it gives the detailed explanation of its application.

Keywords: Nanorobotics, Surgery, cancer therapy, Nanomedicine

\section{INTRODUCTION}

Nanomedicine is the process of treating, diagnosing and preventing disease. It is also used for relieving pain, traumatic injury, for preserving health of human and to improving human health. Nanorobots can be used in various fields to develop and implement the industrial robots and also service robots. Nanorobots are very tiny so they can travel easily throughout the human body. In human body navigational network is installed to monitor the location and activities of human. Robots are used initially to paint the car and also to place the test probes on all electronic circuits. Nanorobotics plays important role in bio medicine. Now day's robots are used in public spaces likeoffices, our homes, vacuum cleaners, lawn mowers, surgical operators, window washers, toys, self-governing airpurifiers etc and also in museums. The nanorobot can be implemented using some devices like power, actuators, sensors, communication, control and some interfacing crossspecial scales. The nanorobots can be developed using some various approaches like Biochip, Nubots, Positional Nanoassembly and Usage of Bacteria. It takes main role in dental applications of robotic dentistry in future with new field is called as Nanorobotics in dentistry. The size of operation can be reduced using the development of Nano technology. Nano robots provide new treatments for patients. In medical field it increases the complexity. Lung cancer is one of the most common cancer 
among all other cancer to cause the death. Nearly $60 \%$ of cancer affected people are treated by radiotherapy. Radiotherapy use ionizing radiation to treat the cancer cells and also other diseases. The ionizing radiation damage DNA structure and affect the growth of DNA. Radiotherapy provide high dosage to affected cells which damage the nearby healthy cells. Intelligent machines have applications like medicine, space, defence, underwater exploration, disaster relief, service industries and entertainment. Sometimes nanorobots are embedded with chemical biosensors to find the tumour cells in early stage of development in the human body.

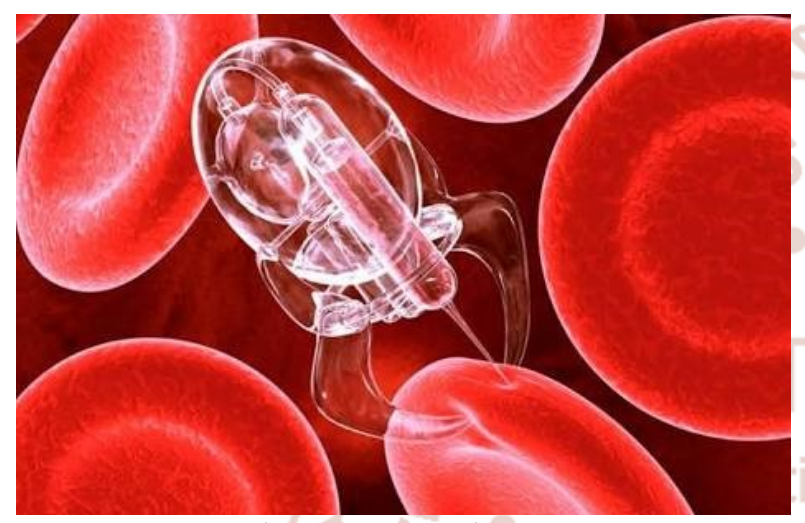

Fig 1: Nanorobots

\section{WORKING OF NANOROBOTS IN CANCER TREATMENT}

To diagnose diseases the nanorobots is send inside the human body. The infected area is diagnosed and the dose of medicine is provided directly to infected area. Two signals are used by nanorobots to treat the affected areas. One is sound wave and other is acoustic signal. The robots receive the power or reprogramming instruction from some external sources and the response is given through acoustic signal. Using these signals the nanorobots is placed in the human body. To treat the cancer cells it should be diagnosed early. The traditional methods like chemotherapy and radiotherapy kills the healthy cells with the cancer cells. Those healthy cells lead to depression, hair loss, fatigue and nausea. Instead of using chemotherapy doctors prefer nanorobots to treat the cancer cells. Nanorobots check the entire body and destroy the cancer cells without producing any harm to healthy cells.

\section{APPLICATIONS OF NANOROBOTS} 1. NANOROBOTICS IN SURGERY

Nanorobots are used for surgery in human body. Those robots are introduced through vascular system and other cavities into the human body. These nanorobots are called as surgical nanorobots. Surgical nanorobots act as a surgeon inside the human body. Surgical Nanorobots act as semi-autonomous on-site in the human body. It is similar to human surgeon. These surgical nanorobots are programmed by human surgeon. The surgical nanorobots perform various functions like diagnosis of diseases, search for pathogens, ultrasonic signals used to contact the supervisory surgeon and so on. The formation of cellular nano surgery can be explored in early stage.

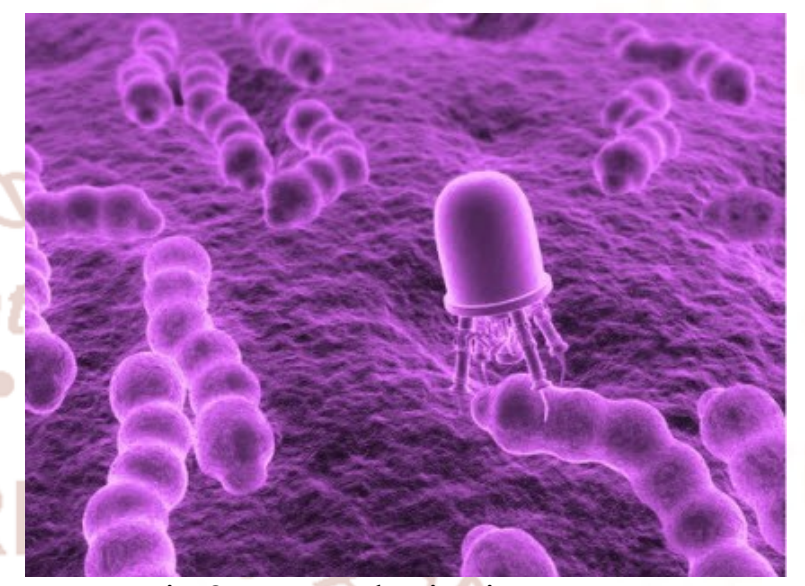

Fig 2: Nanorobotics in Surgery

\section{DIAGNOSIS AND TESTING}

In the human body there are many microorganism, cells, tissues and tumours. These can be monitored, diagnosed and tested using medical nanorobots. Medical nanorobots are used to report with some sign of temperature, pressure and also monitor the immune systems continuously.

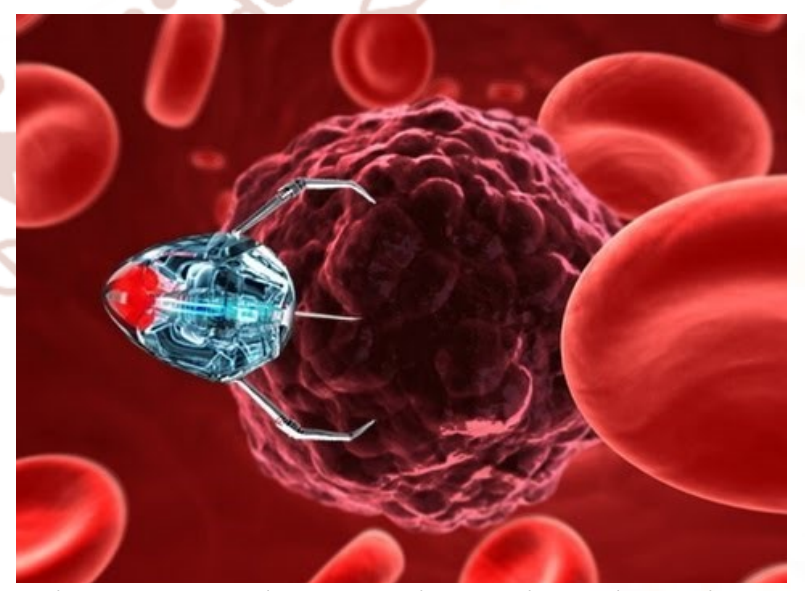

Fig 3: Nanorobots to Diagnosis and Testing

\section{NANOROBOTICS IN GENE THERAPY}

Nanorobots are taking vital role in treating the genetic diseases. It relates the molecular structure of DNA with protein in the cell. There are any modifications or some irregularities takes place in DNA can be corrected. While comparing to cell repair the 
chromosomal replacement is very efficient. The assembled repair vessels is built in the human body to maintain the performance of genetics by floating in the nucleus of cells. Using the robot lower arm the DNA can be enlarged. The upper arms detach the chains protein. That information are stored in database of nano computers which is placed outside the nucleus. The structure of DNA is compared with proteins to repair the cells.

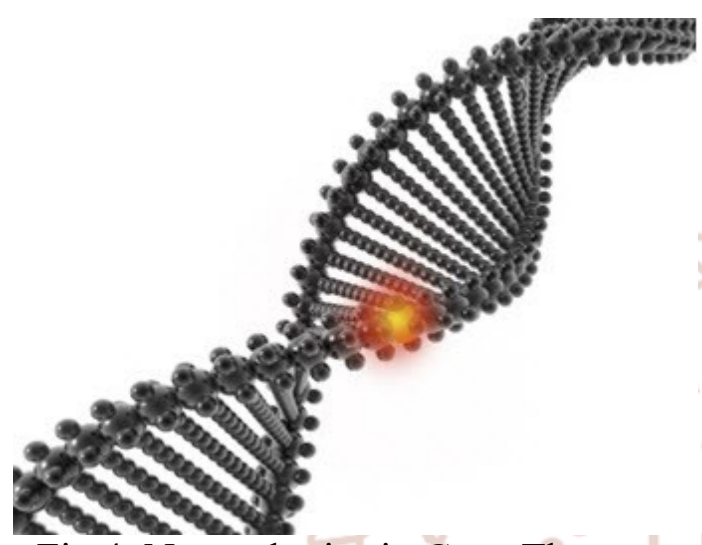

Fig 4: Nanorobotics in Gene Therapy

\section{NANOROBOTS IN CANCER DETECTION AND TREATMENT}

In current stage the nanorobots are used for successful treatment of cancer. The side effect can be decreased by successful treatment of nanorobots. The successful treatment is provided by producing efficient drug delivery. The chemotherapy, radiotherapy and thermal- therapy produce side effects. Chemotherapy use chemicals while providing the treatment which kill the driving cells. The insulin is injected inside the human body, the glucose is monitored using the chemo sensor by nanorobots. Nanorobots is made up of proteins and polymer which is known as transferring which has the capacity to monitor the tumour cells. Nanorobots kill only the cancer cells without affecting the healthy cells.

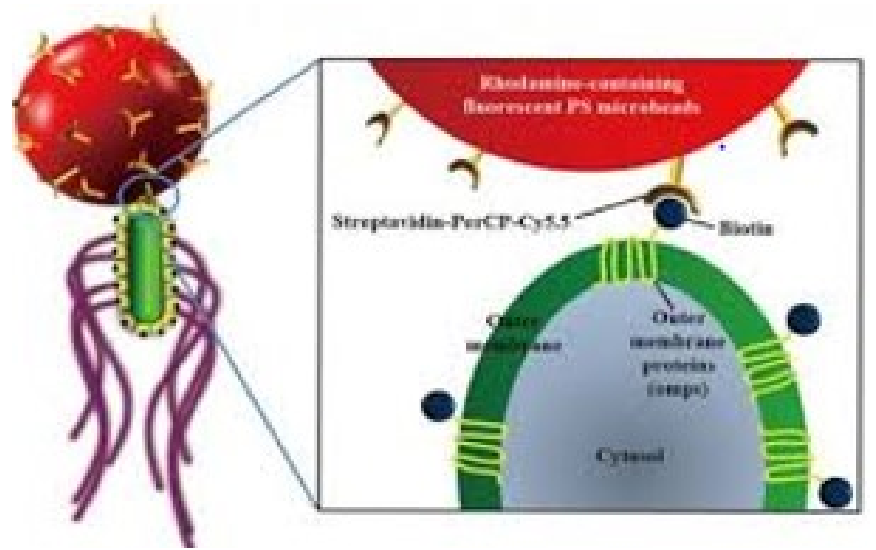

Fig 5: Nanorobots in Cancer Detection and Treatment

\section{CONCLUSION}

Robotic technology is very attractive, challenging and impressive technology. Nanorobots are upcoming technology by interconnecting various areas of science. Cancer takes first place in effect of human life and also the cost of economy. All other traditional system produce many side effects like hair loss, nausea, anaemia, fatigue and so on. Some traditional systems like chemotherapy, radiotherapy affect the healthy cells but nanorobots detect only the cancer cells and protect the healthy cells. Nanorobots detect the cancer cells in early stage and provide the treatment. It saves the life of human by providing better therapy. The diseases are treated very rapidly. Nanorobotics not only treats the cancer cells but also the disease like diabetes, viral infection and so on. Nano medicine helps to earlier diagnosis, early detection and testing, better therapy and also it make the health care more affordable and effective.

\section{REFERENCE}

1. Bhat, A. S. "Nanobots: the future of medicine." Int J Manage Eng Sci 5.1 (2014): 44-49.

2. Yadav, Anurag, Amit Verma, and Kusum Yadav. "Fungal Nanoparticles: An Emerging Tool in Medical Biology." Advances and Applications Through Fungal Nanobiotechnology. Springer, Cham, 2016. 213-240.

3. Girard, Christian. "Robots Don't Care: Why Bots Won't Reboot Architecture." Critical and Clinical Cartographies: Architecture, Robotics, And Medicine, Philosophy (2017): 123.

4. Cerofolini, Gianfranco, et al. "A surveillance system for early-stage diagnosis of endogenous diseases by swarms of nanobots." Advanced Science Letters 3.4 (2010): 345-352.

5. Sierra, Dannelle P., Nathan A. Weir, and James Frank Jones. A review of research in the field of nanorobotics. No. SAND2005-6808. Sandia National Laboratories, 2005.

6. Cavalcanti, Adriano, et al. "Nanorobot architecture for medical target identification." Nanotechnology $19.1 \quad$ (2007): 015103.

7. Sanchez, Samuel, and Martin Pumera. "Nanorobots: The Ultimate Wireless Self-Propelled Sensing and Actuating Devices." Chemistry-An Asian Journal 4.9 (2009): 1402-1410. 
8. Beasley, Ryan A. "Medical robots: current systems and research directions." Journal of Robotics 2012 (2012).

9. Bhattacharyya, Debnath, et al. "Nanotechnology, big things from a tiny world: a review." Nanotechnology 2.3 (2009): 29-38.

10. Mazumder, Sonal. "Nanorobots: Current state and future perspectives." International Journal of Development Research4.6 (2014).

11. Dong, Lixin, Arunkumar Subramanian, and Bradley J. Nelson. "Carbon nanotubes for nanorobotics." Nano today 2.6 (2007): 12-21.

12. Roco, Mihail C. "Nanoparticles nanotechnology research." Journal Nanoparticle Research 1.1 (1999): 1-6.

13. Sujatha, V., Malathi Suresh, and S. Mahalaxmi. "Nanorobotics-a futuristic approach." SRM Univ J Dent Sci 1 (2010): 86-90.

14. Robert, A. "Medical Nanorobotics: The Long-" Term Goal for Nanomedicine." (2011): 367-92.

15. Freitas Jr, Robert A. "Medical Nanorobotics: The Long-Term Goal for Nanomedicine."

16. Cavalcanti, Adriano, et al. "Nanorobot hardware architecture for medical defense." Sensors 8.5 (2008): 2932-2958.

17. Durairaj, R. B., J. Shanker, and M. Sivasankar. "Nano robots in Bio medical application." Advances in Engineering, Science and Management (ICAESM), 2012 International Conference on. IEEE, 2012.

18. Durgakeri, Bhagyashree S., Damini N. Naik, and S. V. Viraktamath. "Application of Nanorobots in Medical field."

19. Bhargava, Amit. "Nanorobots: Medicine of the future." Short Circuit 1 (1999): 1999.
20. Raj, Arun R., N. G. Vijayalekshmi, and S. Akhila. "Nanorobots-Medicine Of The Future." Pharmacies Global 3.12 (2012): 1.

21. Frist, William H. "Health care in the 21st century." New England Journal of Medicine 352.3 (2005): 267-272.

22. Sahoo, S. K., S. Parveen, and J. J. Panda. "The present and future of nanotechnology in human health care." Nanomedicine: Nanotechnology, Biology and Medicine 3.1 (2007): 20-31.

23. Patil, Mallanagouda, Dhoom Singh Mehta, and Sowjanya Guvva. "Future impact of nanotechnology on medicine and dentistry." Journal of Indian society of periodontology 12.2 (2008): 34.

24. Freitas, Robert A. "The future of nanofabrication and molecular scale devices in nanomedicine." Studies in health technology and informatics (2002): 45-60.

25. Freitas Jr, Robert A. "Nanomedicine, Vol. IIA: Biocompatibility." Landes

Georgetown, USA(2003).

26. Kumar, Saravana R., and R. Vijayalakshmi. "Nanotechnology in dentistry." Indian J Dent Res 17.2 (2006): 62-65.

27. Freitas, Robert A. "What is nanomedicine?." Disease-a-month51.6 (2005): 325-341.

28. Cavalcanti, Adriano, et al. "Nanorobot architecture for medical target identification." Nanotechnology 19.1 (2007): 015103.

29. Curtis, Adam S G, Matthew Dalby, and Nikolaj Gadegaard. "Cell signalling arising from nanotopography: implications for nanomedical devices." (2006): 67-72.

30. Patel, Geeta M., et al. "Nanorobot: a versatile tool in nanomedicine." Journal of Drug Targeting 14.2 (2006): 63-67. 